\title{
Childhood disintegrative disorder misdiagnosed as childhood-onset schizophrenia
}

\author{
N S Sawant, MD, DPM, PGDMLS, PGDHHM; S Parker, MD, DPM, PhD; P Kulkarni, MBBS \\ Department of Psychiatry, Seth GSMC and KEM Hospital, Mumbai, Maharashtra, India
}

Corresponding author: N S Sawant (drneenas@yahoo.com)

Childhood disintegrative disorder (CDD) is a rare pervasive developmental disorder, which is often misdiagnosed as schizophrenia, probably due to the resultant severe social impairment and withdrawn behaviour with stereotypys that could be mistaken for psychosis. We report a case of CDD that was misdiagnosed by a psychiatrist as childhood-onset schizophrenia and treated with high doses of antipsychotics. The patient did not show any improvement. This highlights ethical issues that arise from treatment modalities, with polypharmacy being the biggest culprit, and also points to the need to continue medical education at the level of primary health services and among practising rural doctors where tertiary centres with child guidance facilities and a multidisciplinary team are not available.

S Afr J Psych 2014;20(3):94-95. DOI:10.7196/SAJP.518

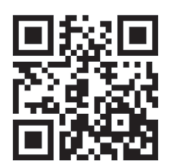

Childhood disintegrative disorder (CDD) is a rare pervasive developmental disorder (PDD) which is characterised by a marked regression in multiple areas of development after 2 years of normal development and before 10 years of age. Its prevalence is around 1 per 100000 children. $^{[1]}$

There is only one reported case of CDD misdiagnosed as schizophrenia, ${ }^{[2]}$ and misattributed diagnoses of psychosis in PDD have been described by Dossetor. ${ }^{[3]}$ We report a case of misdiagnosis in a child with CDD, who was receiving very high doses of antipsychotics.

\section{Case study}

A 12-year-old, boy was brought by parents from a distant village to a tertiary care hospital, with complaints of vomiting, nausea and giddiness, a 6-year history of decreased interaction with family members, sibling and peers, problems in activities of daily living, and inappropriate shouting and laughing.

The patient was a full-term normal delivery with a birth weight of $2.7 \mathrm{~kg}$, and there were no significant antenatal, perinatal or postnatal complications. All his developmental milestones were appropriate until 6 years of age. At this time, his parents realised that the patient was not interacting with his sister and peers, stopped going to play, was self-absorbed and did not demonstrate feelings of attachment or emotion to his parents. His speech had been normal but now deteriorated to only two to three words.

A significant change was also noticed with respect to his ability to bathe, dress, eat, etc. and he had to be helped with all activities. At school the teacher found him to be withdrawn, and he didn't play or do schoolwork. At around the same time, his parents noticed that he was frightened by the photographs of the family deities. He made his parents remove these as he 'felt the eyes of the deity following him. He saw a psychiatrist who diagnosed him with childhood-onset schizophrenia and prescribed oral olanzapine $(10 \mathrm{mg})$, risperidone $(4 \mathrm{mg})$ and trihexyphenydyl $(4 \mathrm{mg})$. While he received the medications, the only improvement was that he slept better. He was promoted to fifth grade at the village school even though he was unable to read or write.

His condition worsened in December 2013, when he started shouting loudly, laughing and crying, and rolling the edges of his shirt and putting them in his mouth throughout the day. He would become irritable and keep on screaming, which caused complaints from the neighbours. He also stopped going to school. His psychiatrist adjusted his medications as follows: oral trifluperazine $(15 \mathrm{mg})$, aripriprazole $(30 \mathrm{mg})$, quetiapine $(50 \mathrm{mg})$, risperidone $(4 \mathrm{mg})$ and trihexyphenydyl $(4 \mathrm{mg})$. Despite being on such high doses for more than a month with hardly any improvement, the psychiatrist advised electroconvulsive therapy for behaviour control. The parents brought the child to Seth GSMC and KEM Hospital for a second opinion.

The child was admitted and examined to rule out organic antecedents before a diagnosis of CDD was considered. He had developed antipsychotic-induced gastrointestinal side-effects, which abated with the stoppage of all the medications. All his blood parameters, magnetic resonance imaging (brain) and electroencephalography were normal. His weight was $25 \mathrm{~kg}$. KayserFleischer rings, markers for Wilson's disease, were not seen and hypothyroidism was ruled out. Ear, nose and throat, ophthalmology and neurology opinions did not reveal any pathology. The child was behaviourally disturbed and was not cooperative during psychological assessment. Oral risperidone $0.5 \mathrm{mg}$ was prescribed after 3 days and titrated to $1 \mathrm{mg}$ in divided doses.

A multidisciplinary team comprising a psychiatric social worker, occupational therapist, psychologist and psychiatrists worked together to educate the parents about $\mathrm{CDD}$, the prognosis and the treatment modalities with sensory integration therapy initiated by the occupational therapist. Occupational therapy observations revealed that 
he had impairment in gross and fine motor skills, including pencil grasp and dexterity. In terms of sensory processing he had problems with auditory registration. Motor planning was affected but direction sense and laterality were intact. The patient required help with daily activities and did not recognise the alphabet, words or numbers. The patient was admitted for 4 weeks, during which gradual improvement was seen in his behaviour, with reduced screaming, shouting and stereotypical movements, and improved eye contact. The patient was from a poor home in a remote village $(500 \mathrm{~km}$ from the hospital) where there were no facilities for continuing sensory integration therapy or a special school. The relatives were counselled about his prognosis and he was prescribed oral piracetam (1 $500 \mathrm{mg})$ in divided doses, to be assessed with respect to improvement in cognitive abilities and understanding in 3 months' time. Surprisingly, at this first follow-up, the parents reported improvement in self-care with self-feeding and self-dressing. The child also managed simple calculations $(2+2,1+3$, etc. $)$, called out to his parents, and at the hospital visit, spoke a short sentence.

\section{Discussion}

There are a few case reports where PDD patients have been misdiagnosed as schizophrenic owing to the severe social impairment and withdrawn behaviour with stereotypys, which could be mistaken for psychosis. ${ }^{[2,3]}$ Studies have reported that early-onset schizophrenia has strong comorbidity with PDD, and there is evidence of accelerated trajectories of anatomic brain development at the ages at which the disorder starts. Furthermore, a growing number of genes and/or rare, small chromosomal variants (microdeletions or duplications) are shared by both the disorders. ${ }^{[4]}$ Studies have found a link between the genes and the chromosomal variants, especially in adult populations and with respect to PDD. ${ }^{[5]}$ However, the management of this patient with such high doses of antipsychotics raises the question of ethical practice and also highlights the difficulties around effective treatment that are faced by the treating clinician. The fact that, in this case, clinical improvement was marginal should have prompted the treating psychiatrist to review the diagnosis to avoid placing the patient at a high risk of developing neuroleptic side-effects.

A detailed history noting all developmental milestones, which help to differentiate CDD/PDD from a psychotic condition, is vital. The self-absorbed behaviour of an autistic child is very different from the avolition or negative features of schizophrenia. Schizophrenia in childhood and adolescence is diagnosed using the same criteria as in adults, but with some minor differences. ${ }^{[1]}$ The variations in clinical presentation can be obvious or subtle, potentially leading to diagnostic confusion. The patient had an apparently normal development till the age of 6 years, and he was a normal, playful, intelligent child attending school. Loss of function was seen particularly in language, social skills, play and motor skills, which prompted consideration of the diagnosis of CDD. Although formal cognitive and educational testing to identify the child's strengths and limitations could not be done, the child did seem to have intellectual impairment, as evidenced by his inability to do simple mathematical calculations, which he was able to do previously, and deterioration in self-care.

Presenting the diagnosis to the family was difficult and they were devastated by the prognosis. They had tried everything for their child, from faith healing to regular treatment from the psychiatrist. Hence it became our duty to provide information, offer support and emphasise the facilities available for these disorders. As the patient was staying in a distant village, there were no experts nearby for continuing the sensory integration, speech therapy or remedial education. Hence, the parents were advised to continue the village schooling simply to improve social interaction.

In terms of medication, risperidone has been the drug of choice for behaviour control in PDD. Piracetam was considered as an adjuvant. Piracetam (2-oxo-l-pyrrolidine) is a cyclic derivative of gammaaminobutyric acid (GABA), which has been used for various cognitive disorders of children as it is associated with increased brain tissue oxygen consumption and an ability to increase the inhibitory hyperpolarising processes in a manner similar to that of GABA. ${ }^{[6]}$

We hoped to see some cognitive improvement over 8 to 10 weeks, and, remarkably, the parents reported improvement within 4 weeks in the boy's understanding and self-care, although social interaction was still minimal. It was heartening to see them happy with their child's progress in basic faculties. We do not know if piracetam has helped the child but at present we are continuing the treatment.

This case highlights the fact that in developing countries, where there are insufficient mental health professionals, it is important to integrate mental health into primary healthcare services, as championed by the World Health Organization through the Mental Health Gap Action Programme.

\section{References}

1. American Psychiatric Association. Diagnostic and Statistical Manual of Mental Disorders. 4th ed. (text revision). Arlington: American Psychiatric Association, 2000.

2. Clarke DJ, LittleJohns CS, Corbett JA, et al. Pervasive developmental disorders and psychoses in adult life. Br J Psychiatry 1989;155:692-699.

3. Dossetor DR. All that glitters is not gold: Misdiagnosis of psychosis in pervasive developmental disorders--a case series. Clin Child Psychol Psychiatry 2007;12(4):537-48. [http://dx.doi.org/10.1177/1359104507078476]

4. Rapoport J, Chavez A, Greenstein D, et al. Autism-spectrum disorders and childhood onset schizophrenia: Clinical and biological contributions to a relationship revisited. Am Acad Child Adolesc Psychiatry 2009;48(1):10-18. [http://dx.doi.org/10.1097/CHI.0b013e31818blc63]

5. Sprong M, Becker HE, Schothorst PF, et al. Pathways to psychosis: A comparison of the pervasive developmental disorder subtype Multiple Complex Developmental Disorder and the 'At Risk Mental State'. Schizophr Res 2008;99(13):38-47. [http://dx.doi.org/10.1016/j. schres.2007.10.031]

6. Winnicka K, Tomasiak M, Bielawska A. Piracetam: An old drug with novel properties? Acta Pol Pharm 2005;62(5):405-409. 